\title{
Introduction. La construction d'une France littéraire : ancrage, réception et création littéraires
}

Introduction. Literary France in the making: literary anchoring, reception and creation

Mathilde Labbé

\section{OpenEdition}

\section{Journals}

Édition électronique

URL : https://journals.openedition.org/recherchestravaux/1906

DOI : 10.4000/recherchestravaux.1906

ISSN : 1969-6434

Éditeur

UGA Éditions/Université Grenoble Alpes

Édition imprimée

ISBN : 978-2-37747-197-3

ISSN : 0151-1874

\section{Référence électronique}

Mathilde Labbé, «Introduction. La construction d'une France littéraire : ancrage, réception et création littéraires », Recherches \& Travaux [En ligne], 96 | 2020, mis en ligne le 23 juin 2020, consulté le 29 juin 2021. URL : http://journals.openedition.org/recherchestravaux/1906 ; DOI : https://doi.org/10.4000/ recherchestravaux.1906

Ce document a été généré automatiquement le 29 juin 2021

(C) Recherches \& Travaux 


\section{Introduction. La construction d'une France littéraire : ancrage, réception et création littéraires}

Introduction. Literary France in the making: literary anchoring, reception and creation

Mathilde Labbé

\section{NOTE DE L'AUTEUR}

Le présent volume a été conçu et réalisé dans le cadre du programme LITEP <http:// monumentslitteraires.com/about-litep-literature-in-the-public-space>, avec le soutien du RFI Tourisme et du GIS Études touristiques.

1 La forme d'une ville, pour les marcheurs que sont Baudelaire, Julien Gracq ou Jacques Roubaud et bien d'autres, ne saurait se résumer aux représentations graphiques que l'on peut en donner. Reprenant un vers du "Cygne ", rêverie urbaine nourrie par le souvenir de Victor Hugo, les deux recueils de pérégrinations publiés par Gracq et Roubaud, La forme d'une ville et La forme d'une ville change plus vite hélas que le cour des humains ${ }^{1}$, sont de beaux exemples de la manière dont la littérature s'invite dans la perception de l'espace urbain. Baudelaire, Balzac, Rimbaud, Queneau: les souvenirs littéraires s'imposent comme un filtre dans la promenade. Mais les écrivains ne sont pas les seuls à se laisser ainsi guider par leurs pairs : Maurice Halbwachs, dans son étude sur la mémoire collective, évoque une promenade faite dans Londres « avec Dickens ${ }^{2}$ ». Par sa capacité à mettre l'espace en récit, la littérature constitue en effet un outil privilégié de médiation aux lieux, ce que les auteurs de guides touristiques ont très tôt repéré comme une niche éditoriale intéressante. Des récits de voyages, guides littéraires et livres de prix de la fin du XIX ${ }^{\mathrm{e}}$ siècle (Frances Miltoun ${ }^{3}$, Jean-Louis Croze $\left.{ }^{4} . ..\right)$ aux volumes publiés de nos jours entre autres par les éditions Alexandrines ${ }^{5}$, éditeurs et professionnels du tourisme s'emploient à reconstituer, ville par ville, région par 
région, une France littéraire. Chaque localité se voit alors attribuer - n'en déplaise à la capitale - une plume célèbre, ce qui permet de démontrer, s'il était nécessaire, la richesse culturelle d'un pays et de ses territoires. Cette "France littéraire ${ }^{6}$ ", cette «France des écrivains ${ }^{7}$ », avatar intellectuel et esthétique d'une déclinaison permettant ailleurs de recenser châteaux, cathédrales et vins ${ }^{8}$, se fonde sur la croyance en une innutrition des œuvres par le cadre de vie de l'écrivain, en un ancrage territorial de la littérature. Elle se nourrit à la fois du mouvement de la littérature régionaliste, de son exploitation éditoriale ${ }^{9}$ et de la mode des promenades littéraires développées par des sociétés savantes tout au long $\mathrm{du} \mathrm{xx}^{\mathrm{e}}$ siècle. Certes, ce jeu d'association a tôt fait de rencontrer ses limites : celle de l'instrumentalisation, d'abord, qui fait du nom d'auteur seul un lien aux lieux, et passe l'œuvre sous silence; celle de la lecture purement référentielle ou d'identification, par ailleurs, qui néglige la dimension créatrice de la littérature ; celle de la lecture biographique, enfin, qui surdétermine le texte par son contexte au mépris de sa dimension symbolique. Il n'en est pas moins persistant, en vertu de la "vérité fictionnelle et performative de toute conversion patrimoniale ${ }^{10}$ ", pour reprendre les termes de Daniel Fabre. Ainsi a-t-on longtemps voulu croire que Savinien de Cyrano, dit de Bergerac, était le représentant idéal de la Dordogne: la lumière a été faite sur l'origine francilienne de son nom mais la ville de Bergerac, qui compte deux statues à l'effigie du personnage dramatique - plutôt que de l'écrivain, à dire vrai-, n'en continue pas moins d'exploiter le mythe authentifié par Rostand et avant lui par Nodier ${ }^{11}$.

Fig. 1

Dessin du château de Combourg illustrant le chapitre consacré à Chateaubriand dans J.-L. Croze, À travers la France Littéraire. Racine, Corneille, Boileau, Mme de Sévigné, Bossuet, Jean-Jacques Rousseau, Chateaubriand, Balzac, Paris, Librairie Felix Juven, 1904, p. 175. Droits réservés.

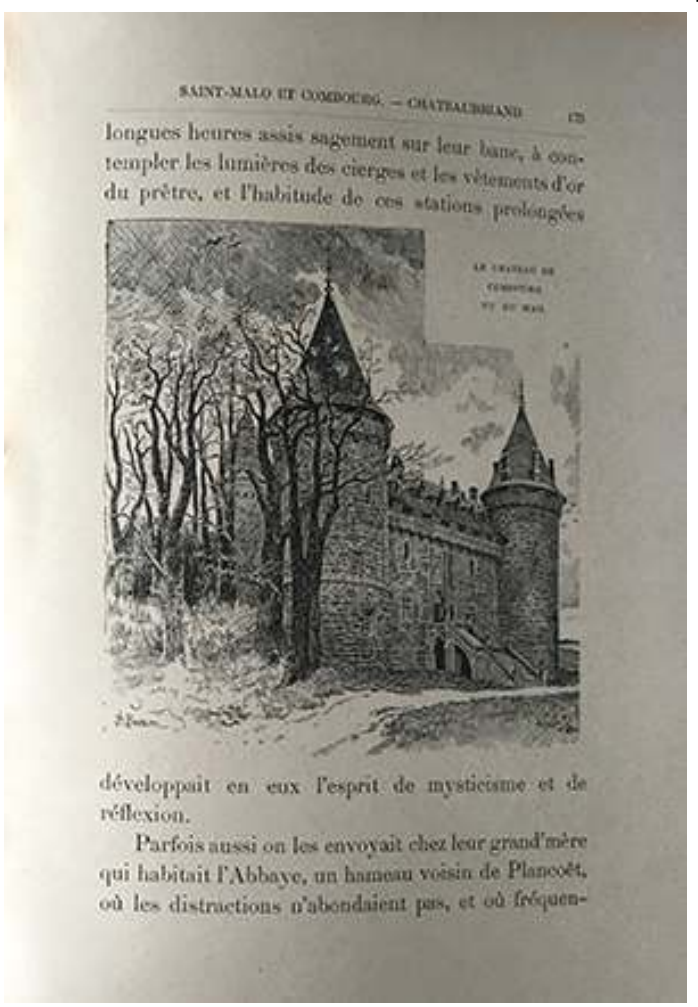


2 La notion d'ancrage permet d'éclairer la manière dont la littérature est conduite à sortir du livre et à devenir une ressource territoriale, de même que le territoire s'invite parfois dans la création littéraire en tant que ressource éditoriale ${ }^{12}$, et d'analyser les fonctions de ces associations œuvres-lieux tant dans la promotion de la littérature que dans celle des territoires. Par ancrage territorial, on entend donc le lien des œuvres et des auteurs aux lieux, que celui-ci soit rendu sensible par le texte ou qu'il résulte de l'appropriation de figures auctoriales par des communautés de lecteurs situées. Considérant cet ancrage de manière pragmatique, on s'intéresse ici autant aux cas consensuels qu'aux cas conflictuels : l'ancrage territorial de la littérature participant de sa patrimonialisation ${ }^{13}$ et de sa trivialisation ${ }^{14}$, il engage à ce titre des conflits de légitimité. Cependant, que les liens entre œuvres et lieux soient ou non souhaités par les écrivains, ils tendent à s'imposer comme grilles de lecture des textes tout comme les textes s'imposent à la mémoire du promeneur. Qu'ils y souscrivent ou non, les écrivains sont d'ailleurs les premiers sollicités dans les campagnes commémoratives ${ }^{15}$. Nombreux sont ceux qui participent avec réticence à ces cérémonies mais qui les soutiennent parce qu'ils y voient un moyen d'assurer par le bas la perpétuation de la mémoire de l'auteur concerné ou des lettres en général, dans un contexte de menaces supposées ou réelles pesant sur le prestige et l'autonomie de la littérature. En témoigne le très riche corpus de discours d'inauguration conservés par l'Académie française ${ }^{16}$.

3 Si la question des rapports entre territoires et littérature a été explorée depuis les années 1970 et le tournant spatial ${ }^{17}$ par la géopoétique, la géocritique puis la géographie littéraire ${ }^{18}$, ces approches ont plutôt privilégié la présence des lieux dans les œuvres et la manière dont celles-ci les transfigurent. Notre dossier analyse, au contraire, la façon dont le rapport aux lieux influe sur la réception des textes, à travers un double phénomène de trivialisation et de patrimonialisation, et, dans certains cas, sur le contexte de leur commande. Seront ainsi explorées les relations entre constructions mémorielles et écriture de l'histoire littéraire; pour cela, nous prendrons appui sur l'étude des lieux de mémoire ${ }^{19}$ et du phénomène commémoratif ${ }^{20}$, tout en combinant approche par les auteurs et approche par les acteurs de cette territorialisation. Il s'agit également de contribuer à l'analyse du tourisme littéraire, jusqu'ici plutôt développée avec les outils de la géographie ${ }^{21}$, via une étude de la circulation des textes et des figures d'auteurs. En envisageant l'ancrage territorial de la littérature, on cherche ici à expliquer ce que l'ancrage fait à l'image des écrivains et au canon littéraire, mais aussi à identifier les acteurs de cette appropriation des textes et des figures d'auteurs, et à analyser, enfin, la relation qui s'instaure alors entre le lecteur et le texte, que celle-ci relève de la lecture savante, de la curiosité biographique ou de la recherche dilettante d'un loisir cultivé. 
Fig. 2

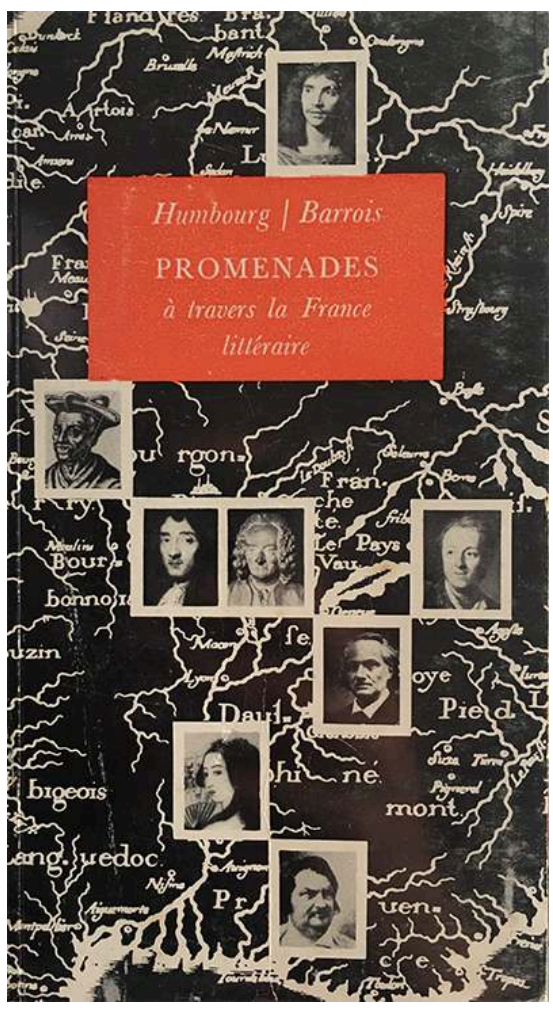

P. Humbourg et M. Barrois, Promenade à travers la France littéraire. Dessins de Gérard Dorville, Paris, Denoël, 1960, couverture. Droits réservés.

Que fait l'ancrage territorial à l'image de l'écrivain? À la suite des travaux sur les rapports entre narrations auctoriales et espace public ${ }^{22}$, ce dossier entend analyser les modifications de ces narrations lors ou en vue d'une actualisation de tel ou tel ancrage. S'il existe un bénéfice au "capital d'autochtonie» pour les écrivains vivants «peu reconnus $^{23}$ ", qui trouvent dans les publications liées au territoire une source de visibilité, qu'en est-il de ce capital pour les écrivains patrimoniaux? Par ailleurs, si plusieurs ancrages régionaux peuvent coexister pour un même écrivain, comme dans le cas de Jules Verne ${ }^{24}$ par exemple, l'articulation entre reconnaissance locale et reconnaissance nationale peut varier de l'un à l'autre. Elle engage également plusieurs niveaux ou cercles de reconnaissance, des pairs à la critique, à l'enseignement et au grand public. Selon quels critères et selon quelle articulation à ces cercles évolue-telle? Enfin, dans la mesure où l'ancrage induit une relation d'appropriation, réciproque ou non, entre l'écrivain et le territoire, on peut se demander quels sont les risques pour les écrivains, en termes d'image mais aussi en termes de liberté dans la création, d'une appropriation locale.

Comment se configurent les rapports entre champ littéraire et champ politique dans cet aller-retour entre le local et le national ? Comment les institutions littéraires, les académies, les sociétés savantes et les autres communautés de lecteurs se saisissentelles de la figure d'un écrivain pour la construction de leur image propre et le développement de leur attractivité touristique ${ }^{25}$ ? Comme le remarque plaisamment Jean Nivet, presque tous les écrivains sont susceptibles d'être mobilisés dans le cadre de la promotion d'un territoire, y compris « un poète comme Racan, que plus personne ne lit ${ }^{26}$ ». Il s'agira d'éclairer la manière dont s'enchevêtrent dans ces phénomènes 
d'appropriation des motivations multiples: celles des auteurs, mais aussi celles des lecteurs privés, des collectivités territoriales ou des associations soutenant la création littéraire et la lecture. Comment se combinent ces différentes dynamiques collectives? Quel rôle joue ou peut jouer la recherche scientifique dans ces combinaisons? On cherchera ainsi à replacer le tourisme littéraire dans le temps long d'une appropriation des auteurs et des œuvres par les lecteurs en s'attachant à l'étude de « communautés interprétatives ${ }^{27}$ » mobilisées autour d'événements littéraires comme les résidences, les lectures et les festivals, ou de commémorations de différentes natures (musées, archives, monuments, plaques commémoratives, noms de rues, noms de bâtiments publics, pèlerinages, cérémonies diverses ${ }^{28}$ - chacune de ces modalités de la commémoration ayant ses spécificités propres). À la diversité des modalités de présence de l'écrivain dans l'espace public s'ajoute la diversité des genres littéraires et critiques susceptibles de nourrir l'étude de ces rapports entre territoires et écrivains : portraits de pays $^{29}$, textes de résidence ${ }^{30}$, correspondance, journaux intimes, promenades littéraires, documents touristiques...

Comment articuler, enfin, le soupçon de fétichisme ${ }^{31}$ pesant sur les manifestations de la gloire littéraire et la possibilité d'une relation critique hors de la lecture au sens strict, reposant sur la dimension discursive d'objets non uniquement textuels? L'apport capital des sciences de l'information et de la communication à l'étude du fait littéraire sera mobilisé pour l'étude de la muséographie ${ }^{32}$, des dispositifs éditoriaux ${ }^{33}$ et des médiations du texte en général. La dimension critique du portrait d'écrivain, qu'il soit sculpté $^{34}$ ou photographié ${ }^{35}$, a été bien établie. Le buste de Jules Verne à Nantes représente un auteur pour enfants, conformément à la réception d'époque, de même que celui de Baudelaire au jardin du Luxembourg ${ }^{36}$ représente un auteur catholique : les deux figures d'auteurs ont évolué par la suite et relégué ces lectures au rang de réceptions situées. Les maisons d'écrivains peuvent être abordées de la même manière : Jean-Paul Dekiss va jusqu'à considérer qu'elles peuvent être conçues comme des outils critiques $^{37}$. Il convient également de reconnaître au pèlerinage et à la curiosité leur capacité à donner lieu, comme l'affirme Jean Nivet, à de véritables "promenades

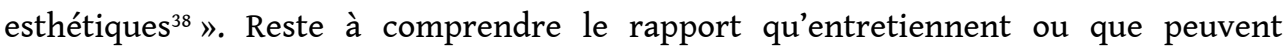
entretenir ces objets avec l'histoire de la critique et l'histoire de l'enseignement de la littérature. L'ancrage de la littérature dans les territoires constitue un mode de lecture des œuvres fortement situé, comportant de ce fait d'évidentes limites, mais dont la vigueur ne se dément pas: loin d'une lecture purement esthétique et universaliste, cette réception décentralisée perdure parce qu'elle se nourrit à la fois du travail ancien des sociétés savantes locales, du besoin renouvelé d'identités territoriales, et, plus récemment, de la revendication d'appropriations non savantes encouragée par la dynamique de démocratisation culturelle du second $\mathrm{xx}^{\mathrm{e}}$ siècle. 
Fig. 3

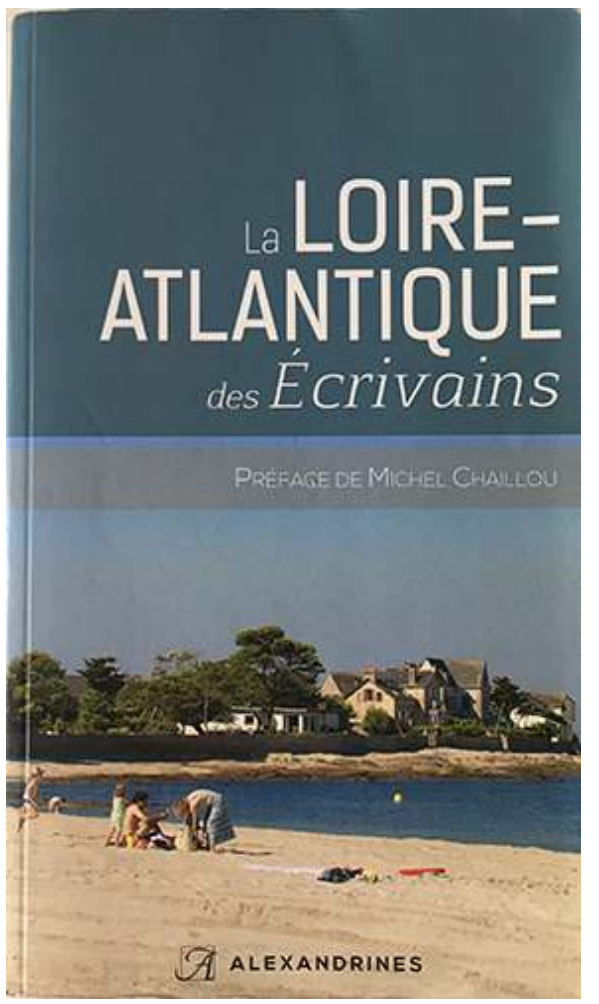

Guide consacré à la Loire-Atlantique dans la collection « La France des écrivains », nouveau de nom de la série "Sur les pas des écrivains ». La Loire-Atlantique des écrivains, préface de Michel Chaillou, Paris, Alexandrines, 2014. Droits réservés.

7 La première section de ce dossier est consacrée aux rapports entre l'ancrage des auteurs dans les territoires et l'évolution ou la formation de leur image. Nathalie Grande s'intéresse ainsi aux patrimonialisations comparées de deux autrices et aux critères selon lesquels celles-ci s'effectuent, en mettant en lumière la différence entre réception scolaire ou universitaire et patrimonialisation. MarieClémence Régnier s'attache pour sa part à la patrimonialisation d'un auteur qui ne faisait pas mystère de son hostilité aux commémorations : Flaubert. Mettant en valeur le caractère aporétique des célébrations rouennaises et parisiennes, elle articule la formation de la mémoire collective à une histoire de la réception critique.

Dans un second temps, nous nous intéressons à la manière dont l'ancrage est mis en œuvre dans différents territoires, pour les écrivains vivants comme pour ceux qui sont déjà entrés dans le canon. Le rôle des résidences dans cet ancrage est capital, tant pour les uns que pour les autres. Dans son article, Anne Reverseau s'interroge sur la manière dont les écrivains contemporains vivent l'ancrage opéré par la résidence, qu'elle compare à une « greffe » de l'écrivain sur le territoire. Elle analyse ainsi le processus de valorisation territoriale et ce que le risque d'instrumentalisation fait à la création littéraire. Les maisons d'écrivain sont un lieu privilégié pour cette territorialisation, comme le montre Carole Bisenius-Penin dans son enquête sur les résidences d'écriture installées dans le cadre de maisons patrimoniales. Elle détaille le double ancrage opéré par ces espaces d'identification et d'appropriation, qui combinent patrimoine matériel et patrimoine immatériel. 
9 La troisième section du dossier combine approche ethnographique et approche historique du tourisme littéraire. Les territoires sont visités sous l'égide de l'écrivain ou de ses personnages, qui informent la perception de l'espace et deviennent médiateurs des lieux. Jean-Marie Privat revient pour cette occasion au village de Ry pour une étude de la patrimonialisation dans ce site littéraire paradoxal : l'appropriation, ici, ignore la textualité du roman mis en tourisme, Madame Bovary, mais s'inscrit dans d'anciens dispositifs de créance encore très vivaces. Cet article permet de mettre en évidence le processus d'identification à l'œuvre dans le tourisme littéraire : les promeneurs sont invités à devenir personnages et à se transformer en "performeurs du récit ». L'étude de Tom Williams sur les voyages littéraires de James et Betham Edwards prolonge cette analyse en montrant comment la lecture informe les impressions de voyage et transforme Balzac, pour les lecteurs britanniques, en guide privilégié de la découverte d'une France authentique car provinciale.

10 Dans un dernier temps, deux articles mettent en perspective la patrimonialisation à l'œuvre dans le tourisme littéraire et les enjeux culturels, médiatiques et politiques de cette territorialisation de la littérature. En s'attachant à l'histoire de la Maison de tante Léonie et du musée Curie, Delphine Saurier envisage le tourisme littéraire comme médiation culturelle. Elle montre comment le romancier ou l'Illustre est érigé en figure culturelle, forcément multiple, et analyse les réseaux qui font émerger le patrimoine à travers ces formes ancrées de la littérature. David Martens s'intéresse quant à lui à l'implication d'écrivains dans la création de portraits de pays. Dans ce cadre, les écrivains deviennent les "opérateurs de patrimonialisation" d'une industrie touristique qui se légitime par la culture.

11 L'étude de Marie-Françoise Melmoux-Montaubin vient clore ce dossier par une analyse panoramique des conditions de patrimonialisation de la littérature, de sa territorialisation et de la manière dont cet « empaysement » s'articule ou s'oppose à la vocation universalisante de l'histoire littéraire ou à une consécration interne au champ.

12 À travers ces propositions, on souhaite interroger la manière dont la littérature intervient dans la formation des «liens aux lieux ${ }^{39}$ ", poser la question de la construction située du patrimoine littéraire, et mettre en lumière les dimensions touristique, éditoriale, urbanistique et sociale de ce phénomène d'ancrage. 
Fig. 4

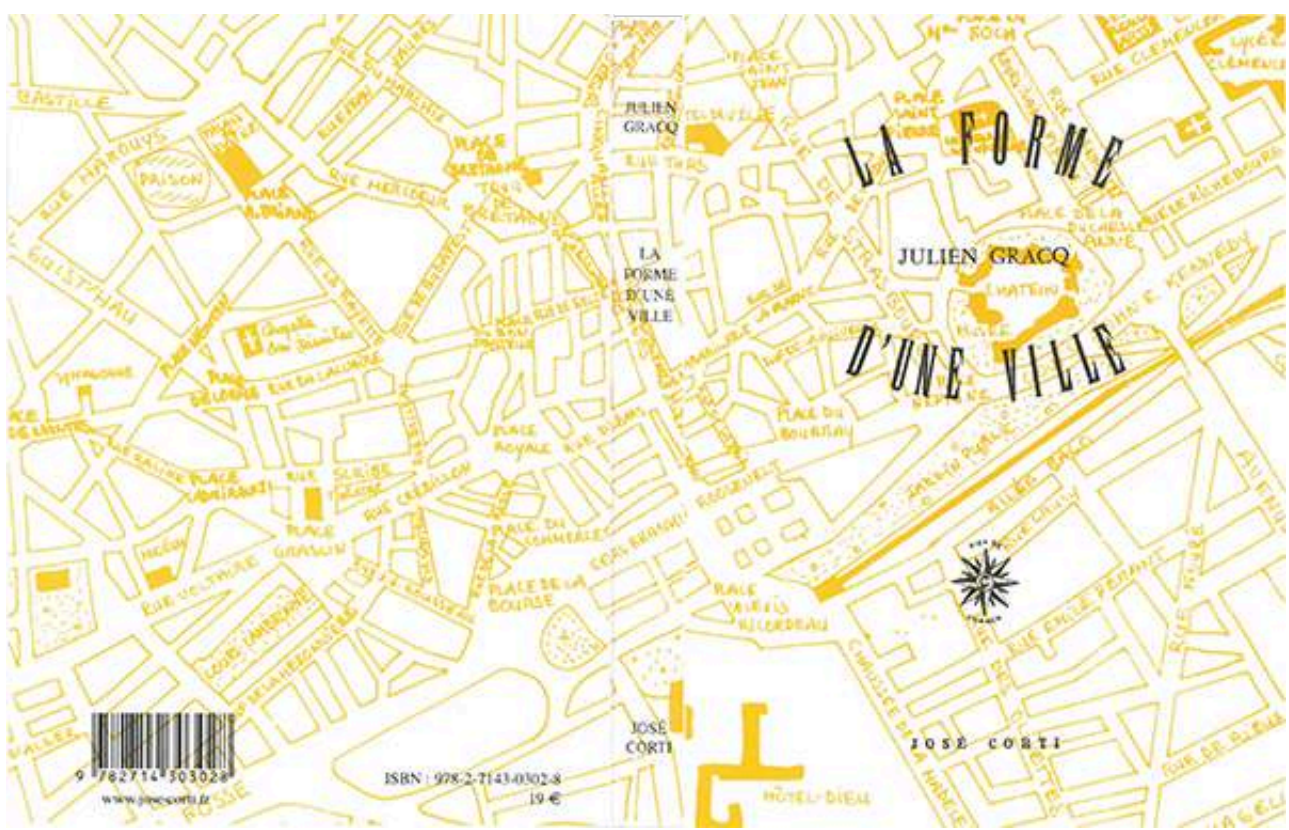

Couverture de La forme d'une ville, ouvr. cité. Plan de Nantes décalqué à la main par Bertrand Fillaudeau pour Julien Gracq et les éditions Corti.

(c) Bertrand Fillaudeau/Corti.

\section{NOTES}

1. C Baudelaire, «Le Cygne», Les Fleurs du Mal [1957], texte établi, présenté et annoté par C. Pichois, Paris, Gallimard, coll. « Bibliothèque de La Pléiade », 1975-1976, t. I, p. 85-87 ; J. Gracq, La forme d'une ville, Paris, José Corti, 1985 ; J. Roubaud, La forme d'une ville change plus vite, hélas, que le cour des humains. Cent cinquante poèmes, Paris, Gallimard, 1999.

2. M. Halbwachs, La mémoire collective [1950], éd. L. Audy \& J.-M. Tremblay, bibliothèque PaulÉmile-Boulet de l'université du Québec à Chicoutimi, coll. « Les classiques des sciences sociales ", 2001, p. 6.

3. F. Miltoun, Dickens' London, Boston, L.C. Page, 1903, et Dumas' Paris, Boston, L.C. Page, 1904 ; A. B. Maurice, The New York of the Novelists, New York, Dodd, Mead and Co., 1915, et The Paris of the Novelists, New York, Doubleday, 1919, p. xx. Voir l'article de Tom Williams « Lire et visiter la France de Balzac » dans le présent numéro (Recherches \& Travaux, $\mathrm{n}^{\circ} 96,2020$ ).

4. J.-L. Croze, À travers la France Littéraire. Racine, Corneille, Boileau, Mme de Sévigné, Bossuet, JeanJacques Rousseau, Chateaubriand, Balzac, Paris, Librairie Felix Juven, 1904.

5. Les éditions Alexandrines, fondées en 1997, se spécialisent dans les guides littéraires et ont créé plusieurs collections sur ce thème telles que « Sur les pas des écrivains » (depuis 1998), « Les écrivains vagabondent» (depuis 2010), «Terres d'écrivains» (depuis 2012) ou "Le Paris des écrivains » (depuis 2015).

6. Par exemple : P. Humbourg et M. Barrois, Promenade à travers la France littéraire. Dessins de Gérard Dorville, Paris, Denoël, 1960. 
7. Voir par exemple les projets «GéoCulture - La France vue par les écrivains " ( GéoCulture : le Limousin vu par les artistes", L'Observatoire, 2010/2, nº 37, p. 82-85, en ligne: <https:// www.cairn.info/revue-1-observatoire-2010-2-page-82.htm>), projet culturel engagé par des réseaux régionaux du livre et de la lecture; «La Lorraine des écrivains " (<http:// lalorrainedesecrivains.univ-lorraine.fr/en/cartographie-auteurs/>), projet de recherche sur les liens entre littérature et territoires ; Ancrages-Passages, web-documentaire consacré aux écrivains de Picardie, 2017 (<http://canalnord.org/ancrages-passages/\#ACCUEIL>).

8. Voir, dans ce numéro, les remarques de M.-F. Melmoux-Montaubin sur les conséquences de la patrimonialisation de la littérature (Recherches \& Travaux, $\mathrm{n}^{\circ}$ 96, 2020).

9. Voir A.-M. Thiesse, «Le mouvement littéraire régionaliste (1900-1945)», Ethnologie française, nouvelle série, t. 18, n 3, Régionalismes, 1988, p. 220-232, et C. Roudeau, «Écritures régionalistes (1800-1914) : nouvelles échelles, nouveaux enjeux critiques », Romantisme, 2018/3, nº 181. Parmi les anthologies exploitant cette veine, voir par exemple A. van Bever, Les poètes du terroir: du $X_{V I}^{e}$ siècle au XXe siècle, 1918, Paris, C. Delagrave, t. I, et Poètes du terroir, recueillis par G. Janet, Dierville, 1942.

10. D. Fabre, « Maison d'écrivain. L'auteur et ses lieux », Le Débat, 2001/3, n 115, p. 176-177.

11. C. Nodier, «Cyrano de Bergerac », Revue de Paris, t. XXIX, 1831, p. 42.

12. G. Bois, «Ancrage local et visibilité littéraire. Le cas des écrivains peu reconnus de la région Rhône-Alpes ", Ethnologie française, 2014/4, vol. 44, p. 621-629.

13. D. Poulot (dir.), Patrimoine et modernité, Paris/Montréal, L'Harmattan, coll. «Chemins de la mémoire ", 1998 ; J. Davallon, Le Don du patrimoine. Une approche communicationnelle de la patrimonialisation, Paris, Lavoisier, coll. "Communication, médiation et construits sociaux", 2006 ; G. Di Meo, « Processus de patrimonialisation et construction des territoires », dans Colloque "Patrimoine et industrie en Poitou-Charentes : connaitre pour valoriser », Poitiers/Châtellerault, Geste éditions, 2008, p. 87-109.

14. Y. Jeanneret, Penser la trivialité, t. I., Paris, Lavoisier, coll. « Hermès science », 2008.

15. Voir le rôle joué par les poètes membres de la Société des poètes français dans les commémorations de leurs pairs et, par exemple, l'implication de Leconte de Lisle, Anatole France, Paul Valéry ou Pierre Emmanuel dans les commémorations de Baudelaire : Mathilde Labbé, «Commémorer le dernier voyage: Baudelaire 2017 », Le Magasin du XIX siècle, $\mathrm{n}^{\circ} 7$, novembre 2017, p. 216-220.

16. Pour une étude de ces discours, voir M. Labbé, «Les monuments de la nation littéraire ", Actes $d u$ colloque narrations auctoriales dans l'espace public, C. Bisenius-Penin et J. Glesener (dir.), Questions de communication, série « Actes », à paraître en 2020.

17. Voir M. Collot, Pour une géographie littéraire, Paris, José Corti, coll. « Les essais », 2014.

18. B. Westphal, La Géocritique, Réel, Fiction, Espace, Paris, Minuit, 2007 ; M. Collot, ouvr. cité.

19. P. Nora (dir.), Les Lieux de mémoire, Paris, Gallimard, coll. «Bibliothèque des histoires ", 1984-1992.

20. J.-M. Goulemot et É. Walter, «Les Centenaires de Voltaire et de Rousseau », dans Les Lieux de mémoire, ouvr. cité, t. I, 1984, p. 381-420 ; S. Le Ray, "Commémorer : pourquoi ? Comment?", Bulletin des bibliothèques de France (BBF), $\mathrm{n}^{\circ} 2$, 2000, p. 100-102; J. Leerssen \& A. Rigney, Commemorating Writers in Nineteenth-Century Europe. Nation-Building and Centenary Fever, London, Palgrave Macmillan, 2014.

21. M. Fournier, et al., «Le tourisme littéraire, lire entre les lieux », Téoros: Revue de recherche en tourisme, vol. 37, $\mathrm{n}^{\circ} 1,2018$; G. Molina, Les Faiseurs de ville et la littérature: lumières sur un starsystem contemporain et ses discours publics, thèse de doctorat, Sciences de l'homme et société, université Toulouse le Mirail - Toulouse II, 2010.

22. C. Bisenius-Penin et J. Glesener (dir.), Narrations auctoriales dans l'espace public : comment penser et raconter l'auteur?, colloque de l'université de Lorraine, mai 2019.

23. G. Bois, art. cité. 
24. M. Labbé, «Ancrage local et concurrence commémorative: le cas de Jules Verne (1905-2005) », dans M.-C. Régnier (dir.), Centenaires, jubilés, commémorations, " toasts ». Les cortèges de la mémoire (XVIII $-\mathrm{XXI}{ }^{e}$ siècles), Paris, SERD, coll. «L'Atelier du XIX siècle », mars 2019. En ligne : <https://f.hypotheses.org/wp-content/blogs.dir/3676/files/2019/03/Labbe.pdf>.

25. Voir M. Fournier, «Les écrivains, une ressource territoriale pour les petites villes? Expériences et limites ", séminaire Tourisme et commémoration littéraires, université de Nantes, 20 novembre 2019.

26. J. Nivet, "Pèlerinages littéraires, promenades esthétiques", dans N. Lavialle et J.B. Puech (dir.), L'Auteur comme cuvre. L'auteur, ses masques, son personnage, sa légende, Presses universitaires d'Orléans, 2000, p. 69-88, ici p. 70.

27. S. Fish, Quand lire c'est faire. L'autorité des communautés interprétatives [1980], trad. É. Dobenesque, Paris, Les Prairies ordinaires, 2007.

28. Sur l'histoire de ces monuments, voir, entre autres M. Agulhon, « Nouveaux propos sur les statues de "grands hommes" au XIX ${ }^{\mathrm{e}}$ siècle ", Romantisme, n 100, 1998, p. 11-16 ; J. E. Hargrove, Les Statues de Paris : la représentation des grands hommes dans les rues et sur les places de Paris, Paris, Albin Michel, 1989 ; D. Milo, «Les noms de rues ", dans Les Lieux de mémoire, ouvr. cité, vol. 3/2, 1986, p. 283-315.

29. A. Reverseau (dir.), Portraits de pays illustrés Un genre phototextuel, La Revue des lettres modernes, série « Lire et voir », n 3, 2017 ; S. Lécole Solnychkine, D. Martens, J.-P. Montier (dir.), Portraits de pays. Textes, images, sons, colloque de Cerisy, juillet 2019.

30. C. Bisenius-Penin (dir.), Résidence d'auteurs, création littéraire et médiations culturelles (1). À la recherche d'une cartographie en Grande Région, Nancy, PUN - Éditions Universitaires de Lorraine, 2015.

31. D. Fabre, art. cité.

32. D. Saurier, La Fabrique des illustres, Paris, Éditions Non standard, 2013.

33. E. Souchier, "Formes et pouvoirs de l'énonciation éditoriale", Communication et langages, $\mathrm{n}^{\circ} 154,2007$, «L'énonciation éditoriale en question », p. 23-38.

34. M. Garval, 'A Dream of Stone': Fame, Vision, and Monumentality in Nineteenth-Century French Literary Culture, Newark, University of Delaware Press, 2004 ; J. Lalouette, « Les écrivains dans la statuaire publique (France. 1803-2017)», dans Centenaires, jubilés, commémorations, ouvr. cité. En ligne : <https://f.hypotheses.org/wp-content/blogs.dir/3676/files/2019/03/Lalouette.pdf>.

35. J.-P. Montier, D. Martens \& A. Reverseau (dir.), L'Écrivain vu par la photographie, Presses universitaires de Rennes, 2017.

36. Pour une histoire de ce buste, voir M. Labbé, Héritages baudelairiens 1931-2013, thèse de doctorat, université Paris-Sorbonne, 2014, en particulier p. 377-395.

37. J.-P. Dekiss, «La maison d'écrivain, utopie ou enjeu de société », Revue d'histoire littéraire de la France, 2009/4, vol.109, p. 783-795. Voir aussi la manière dont Delphine Saurier analyse les dispositifs de médiation que sont les maisons d'Illustres dans le présent volume (Recherches \& Travaux, $\mathrm{n}^{\circ}$ 96, 2020).

38. J. Nivet, art. cité, p. 87.

39. Nous empruntons ce terme à Laurent Devisme, qui a activement participé à la réflexion ayant initié ce dossier. L. Devisme, « Territorialités contrastées. Pour une analyse du versant territorial des identités. Les degrés de liens aux lieux observables à St-Florent-le-Vieil », dans J. Boissonade, L. Devisme, J. Stavo-Debauge, N. Auray, S. Prat, Cultures territoriales et sociabilités dans le mouvement, 2003. <hal-00841522> 


\section{RÉSUMÉS}

Par sa capacité à mettre l'espace en récit, la littérature constitue un outil privilégié de médiation aux lieux, ce que les auteurs de guides touristiques ont très tôt repéré comme une niche éditoriale intéressante. Du XIX ${ }^{e}$ siècle à nos jours, éditeurs et professionnels du tourisme se sont employés à reconstituer une France littéraire, dans laquelle chaque localité se voit attribuer une plume célèbre, permettant de démontrer la richesse culturelle du pays et de ses territoires. Cette «France littéraire» ou «France des écrivains", avatar intellectuel et esthétique d'une déclinaison permettant ailleurs de recenser châteaux, cathédrales et vins, se fonde sur la croyance en une innutrition des œuvres par le cadre de vie de l'écrivain, en un ancrage territorial de la littérature. Certes, ce jeu d'association a tôt fait de rencontrer ses limites: celle de l'instrumentalisation, celle de la lecture référentielle ou celle de la lecture biographique - il n'en est pas moins persistant et souvent performatif. Ce dossier explore l'histoire et les fonctions des associations œuvres-lieux tant dans la promotion de la littérature que dans celle des territoires. Par ancrage territorial, on entend le lien des œuvres et des auteurs aux lieux, que celui-ci soit rendu sensible par le texte ou qu'il résulte de l'appropriation de figures auctoriales par des communautés de lecteurs situées. Cette actualisation conduit la littérature à sortir du livre pour devenir une ressource territoriale, de même que le territoire s'invite parfois dans la création littéraire en tant que ressource éditoriale. Il s'agit de mettre en lumière ce que l'ancrage fait à l'image des écrivains et au canon littéraire, d'identifier les acteurs de cette appropriation des textes et des figures d'auteurs, et d'analyser la relation qui s'instaure alors entre le lecteur, l'auteur et le texte.

Thanks to its ability to create narratives about space, literature constitutes a privileged way of mediation to places. Touristic guide books authors have thus early identified it as an interesting editorial niche. From $19^{\text {th }}$ century onwards, publishers and tourism professionals have tried to reconstitute a Literary France, in which every locality is assigned a famous writer, thus demonstrating the cultural richness of the country and its territories. This "literary France" or "France of writers", an intellectual and aesthetic avatar of a declination otherwise identifying castles, cathedrals and wines, is based on the belief in an innutrition of literary works by the writer's environment, in a territorial anchoring of literature. Admittedly, this association game soon meets its limits: instrumentalization, referential reading or biographical reading-it is nonetheless persistent and often performative. This volume explores the history and functions of works-places associations, both in the promotion of literature and in that of the territories. By territorial anchoring, we mean the link between works or authors and places, whether it is made sensible by the text or is the result of the appropriation of authoritative figures by located communities of readers. This actualization leads literature to become a territorial resource outside of the book, just as the territory sometimes invites itself in literary creation as an editorial resource. We here aim at highlighting what the anchoring of literature does to the writers' image and to the literary canon, at identifying the actors of this appropriation of texts and author figures, and at analyzing the relation which then establishes itself between the reader, the author and the text.

\section{INDEX}

Mots-clés : patrimoine, ancrage territorial, tourisme littéraire, réception, légitimation

Keywords : heritage, territorial anchoring, literary tourism, reader's response, legitimation 


\section{AUTEUR}

\section{MATHILDE LABBÉ}

Mathilde Labbé est maîtresse de conférences en Langue et littérature françaises des XIX et $\mathrm{Xx}^{\mathrm{e}}$ siècles à l'université de Nantes. Ancienne élève de l'École normale supérieure et de l'Institut d'études politiques, elle a soutenu une thèse sur la réception de l'œuvre de Baudelaire au Xx ${ }^{\mathrm{e}}$ siècle (Héritages baudelairiens : 1931-2013, Université Paris-Sorbonne, 2014). Ses recherches portent sur la construction du canon littéraire, la réception (Les XIXe siècles de Roland Barthes, dir. José-Luis Diaz et Mathilde Labbé, Bruxelles, Les Impressions nouvelles, 2019) et la patrimonialisation de la littérature (Une fabrique collective du patrimoine littéraire [XIX ${ }^{e}$-XXI ${ }^{e}$ siècles]. Les collections de monographies illustrées, dir. David Martens et Mathilde Labbé, Mémoires du lire / Studies in Book Culture, vol. 7, $\mathrm{n}^{\circ}$ 1, 2015). Elle dirige actuellement le programme La littérature dans l'espace public (université de Nantes/EC Nantes) sur la place et le sens de la commémoration littéraire dans la ville. 